\title{
Reconstruction of nuclear quadrupole interaction in (In,Ga)As/GaAs quantum dots observed by transmission electron microscopy
}

\author{
P. S. Sokolov and M. Yu. Petrov* \\ Spin Optics Laboratory, Saint Petersburg State University, 198504 St. Petersburg, Russia \\ T. Mehrtens, K. Müller-Caspary, and A. Rosenauer \\ Institut für Festkörperphysik, Universität Bremen, 28359 Bremen, Germany \\ D. Reuter \\ Department Physik, Universität Paderborn, 33098 Paderborn, Germany \\ A. D. Wieck \\ Angewandte Festkörperphysik, Ruhr-Universität Bochum, 44780 Bochum, Germany
}

(Dated: November 3, 2018)

\begin{abstract}
A microscopic study of the individual annealed (In,Ga)As/GaAs quantum dots is done by means of highresolution transmission electron microscopy. The Cauchy-Green strain-tensor component distribution and the chemical composition of the (In,Ga)As alloy are extracted from the microscopy images. The image processing allows for the reconstruction of the strain-induced electric-field gradients at the individual atomic columns extracting thereby the magnitude and asymmetry parameter of the nuclear quadrupole interaction. Nuclear magnetic resonance absorption spectra are analyzed for parallel and transverse mutual orientations of the electricfield gradient and a static magnetic field.

PACS numbers: 78.67.Hc, 78.47.jd, 76.70.Hb, 73.21.La
\end{abstract}

\section{INTRODUCTION}

The spin physics of semiconductors has been developed for bulk materials and demonstrated a wide variety of linear and nonlinear phenomena, realized thanks to the optical orientation..$^{1}$ It has been reborn in semiconductor nanostructures in recent decades. ${ }^{2}$ A significant part of the spin-related phenomena are underlain on the dynamic spin polarization of the nuclear spins being polarized by means of the transfer of the photon angular momentum to the nuclear-spin system via electron-nuclear hyperfine interaction. ${ }^{3,4}$ The achievement of a high polarization of the nuclear-spin system becomes challenging for quantum dot (QD) systems where a single spin of an electron would be strongly localized ${ }^{5}$ and is under the influence of the nuclear spin fluctuations paving the way to a fast carrier-spin relaxation. ${ }^{6-12}$ In spite of a combination of a large variety of methods tried to be used for reaching a sufficiently high degree of nuclear-spin order, ${ }^{13-29}$ the experimental achievement of the spin polarization, close to hundred percent, is still a challenging problem, limited, in some particular cases, by the quantum nature of the spin system. ${ }^{30-33}$ A further microscopic analysis including a combination of experimental methods like nuclear magnetic resonance (NMR) ${ }^{34-37}$ and/or various spin noise measurements ${ }^{38-40}$ in combination with atomistic modeling ${ }^{41,42}$ are highly required to gain insight into such an intriguing problem.

In self-assembled QDs, $N \sim 10^{5}$ nuclear spins interact with the localized-electron spin with different strengths because of a spread of the electron density. However, an additional spread of the interaction exists even at nuclear-spin level due to the crystal lattice deformation caused by built-in strain. This introduces a non-homogeneous nuclear quadrupole interaction, changing the usual nuclear-spin dynamics ${ }^{43}$ but also modifying the NMR spectrum both in the single QDs ${ }^{36}$ and in the QD ensembles. ${ }^{44,45}$ The microscopic analysis of the quadrupole interaction is a rather complex problem while any direct experimental measurement of its magnitude would hardly be realized in practice. Since only scant experimental progress can be expected thereupon, an atomistic analysis would at least give some microscopic information within the framework of a chosen model. ${ }^{42}$

In this paper, we address the investigation of the structural properties of a single QD with respect to the analysis of nuclear quadrupole interaction. We make use of the highangle annular dark-field (HAADF) imaging of an individual self-assembled QD in a scanning transmission electron microscope (STEM). This allows us to resolve a crystal lattice with atomic-column resolution that can be used to determine the shape and chemical composition of the QD. The investigated sample is a heterostructure grown by molecular-beam epitaxy on a GaAs substrate and contains 20 layers of (In, Ga)As QDs, embedded in a GaAs matrix. The post-growth thermal annealing of the structure allows for activation of in-diffusion of Ga atoms inside the QD that reduces the number of structure defects and, in addition, blue shifts the ground-state excitonic transition. ${ }^{46-48}$ The obtained STEM images were postprocessed with geometric phase analysis (GPA), from which the Cauchy-Green strain tensor components are extracted. In addition, to obtain the profile of the In and Ga concentrations inside the QD, the local chemical composition is determined by means of atomically resolved HAADF-STEM and energy-dispersive X-ray (EDX) spectroscopy. These data were then used to reconstruct the distribution of the straininduced electric-field gradients (EFG) causing the nuclear quadrupole interaction. Further calculations allow the inter- 


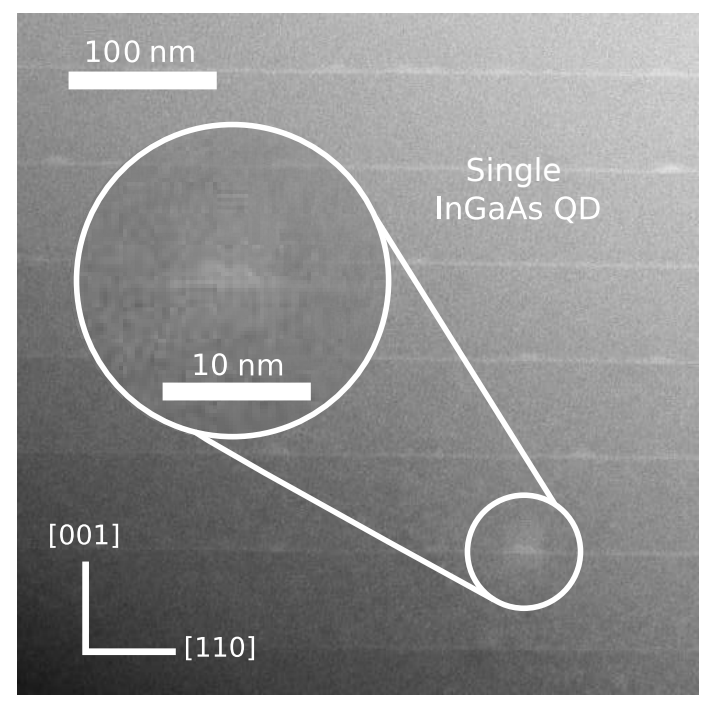

Figure 1. Cross-section micrograph of the heterostructure with annealed (In,Ga)As/GaAs QDs obtained using scanning transmission electron microscopy.

pretation of the NMR transitions that can be detected in the ensemble of such QDs. ${ }^{35,44,45}$

The paper is organized as follows. In Sec. I, we present the details of the microscopic study. The results of the postprocessing of the microscopic images are discussed. In Sec. II, we discuss the possibility of application of microscopic methods for an analysis of strain-induced nuclear quadrupole interaction and quantitative modelling of the NMR spectra. The main results are summarized in Sec. III.

\section{HAADF-STEM IMAGING AND STRAIN MAPPING}

To obtain the crystal strain and, correspondingly, the quadrupole interaction, high-quality microscopic imaging is required ${ }^{49}$ In particular, the image should include the investigated QD and a region of the surrounding GaAs matrix of much larger area than the size of the $\mathrm{QD}$. This region is used as a bulk reference area for further analysis. Selection of the appropriate microscopy method is based on the ability to resolve the atomic columns of the crystal and the ability to extract the chemical composition of the QD. Such data are obtained by analysing the heterostructure with STEM using HAADFdetector, providing information about the concentration of the atoms of different species. ${ }^{50-53}$

The cross-sectional specimens of the sample with an area of about $0.2 \mathrm{~cm}^{2}$ are prepared by mechanical cutting of the heterostructure in the (110) crystallographic plane and then glueing to a tripod holder. The specimens are then mechanically polished reducing the thickness of the sample down to $\sim 10$ $30 \mu \mathrm{m}$. Further ion etching of the QD containing region is applied using a Gatan precision ion polishing system (PIPS) until a hole is etched into the sample. At the edge of this hole the sample thickness is clearly below $200 \mathrm{~nm}$ and thus transparent for electrons. The experiments are performed with an FEI

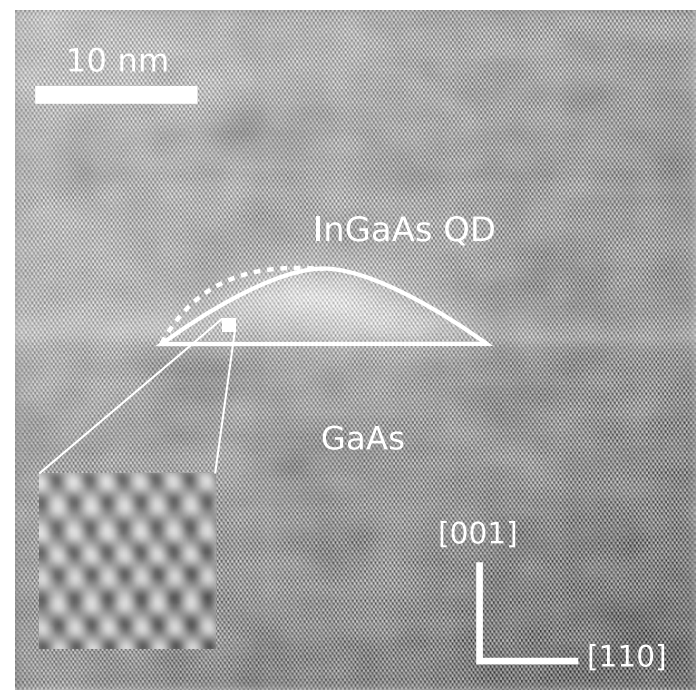

Figure 2. High-resolution STEM image of a single (In,Ga)As QD embedded in the GaAs matrix. The shape of the QD is marked by the thin solid line guide to the eyes with respect to the Indium concentration profile shown in Fig. 3. Atomic-column resolution is demonstrated in the inset.

Titan 80/300 microscope operated at $300 \mathrm{kV}$ electron-beam acceleration voltage and equipped with an image-aberration corrector, EDX, and HAADF detectors. The overview image of the structure is shown in Fig. 1, from which multiple QDs placed on several wetting layers are resolvable. Size and shape of the dots are found to be weakly dispersed, which is also confirmed by a relatively narrow linewidth of the ground-state photoluminescence studied previously with these QDs. ${ }^{46,47} \mathrm{~A}$ few randomly chosen single QDs are analyzed.

Consequently, the high-resolution HAADF-STEM images were recorded in the QD-containing region of the sample. The image size is selected to approximately $50 \mathrm{~nm} \times 50 \mathrm{~nm}$ and the spatial resolution of the microscope is around $0.12 \mathrm{~nm}$ in the scanning mode. Each image contains one QD plus a sufficient amount of the surrounding GaAs matrix, which can be used as reference material for thickness measurement. As high resolution was obtained in the HAADF-STEM images, they were not only suitable for atomic Z-contrast evaluation ${ }^{54-58}$ but could be also used for geometric phase analysis. ${ }^{59,60}$ The chemically sensitive image contrast of the HAADF-STEM images (see Fig. 2) is due to the used HAADF-detector. This ring-shaped detector detects only electrons that are scattered into high-angles (36-220 mrads for the camera length used in this work). The amount of electrons scattered into this region strongly depends on the nuclear charges of the scattering specimen atoms.

The following evaluation procedure was performed for every single image in order to obtain the chemical composition. First, all atomic columns in the high-resolution image were identified. To this end, a Wiener Filter has been applied on the original image. ${ }^{49,61}$ After that, the image has been divided into "Voronoi"-cells and the mean intensity has been calculated for each "Voronoi"-cell and has been assigned to the corresponding atomic column. Note that in this step the inten- 


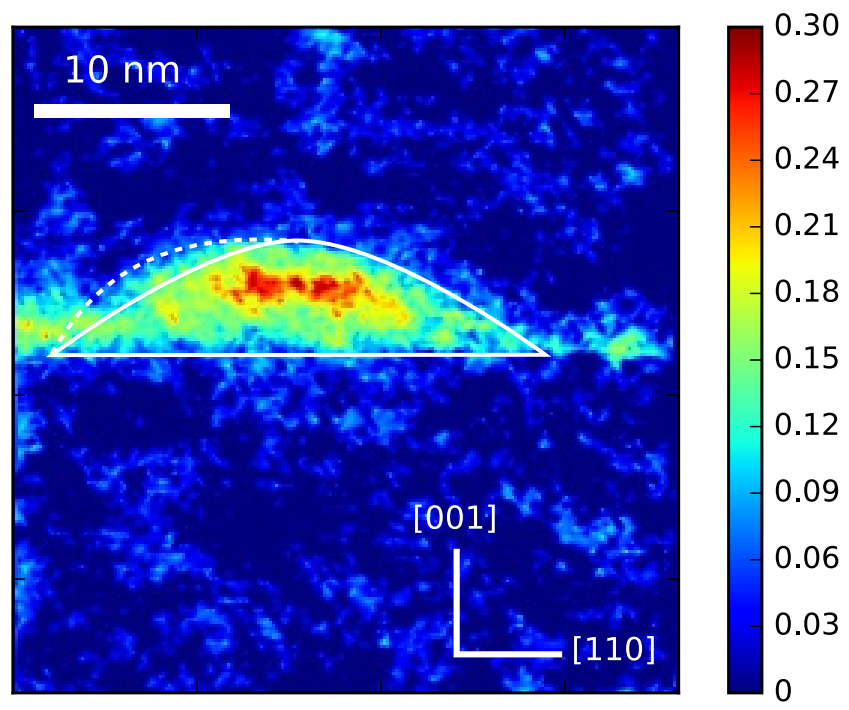

Figure 3. (Color online) Indium concentration map in (In,Ga)As solid compound evaluated from HAADF-STEM. The solid line represents a boundary of the QD surrounding the piece of sample with an In-concentration higher than 0.1 reaching the maximum value 0.3 in the center of the QD.

sities of the original unfiltered image were used. To allow for the quantitative comparison to simulated data, the mean intensity values were normalized with respect to the intensity of the scanning electron probe. In the following step, the normalized intensities were compared to reference data from multislice simulations in the frozen lattice approach using the STEMsim program $^{62}$ carried out in dependence of sample thickness and indium fraction. For more details on the simulations we refer the reader to Ref. 57. Finally, the sample thickness was evaluated from the GaAs region and interpolated over the QD to allow for the determination of the indium concentration as shown in Fig. 3.

To verify the accuracy of HAADF-STEM, EDX spectroscopy is used. The EDX spectra were also acquired in HAADF-STEM mode. While the beam is centred on a small region of the sample surface, the $X$-ray counts were integrated over one minute. Both methods of the Z-contrast evaluation show very similar values for the maximum concentration of Indium in the single QD, i. e., $0.3 \pm 0.05$ and $0.35 \pm 0.05$ for EDX and HAADF-STEM, respectively.

Several methods for mapping the crystal-lattice strain can be applied. ${ }^{59,60,63-65}$ In the electron microscope, none of them is able to evaluate the strain-tensor component along the electron-beam propagation direction. However, the twodimensional maps of two diagonal strain-tensor components could be extracted. For further analysis, the component of shear strain is also required. Denoting the Cartesian coordinate system with respect to crystallographic axes so that $x \leftrightarrow$ [110] and $z \leftrightarrow$ [001], the Cauchy-Green strain-tensor components $\varepsilon_{x x}, \varepsilon_{z z}$, and $\varepsilon_{x z}$ are obtained. While other components are experimentally unaccessible, the symmetry of the problem allows us to equalize the $x$ and $y$ directions, keeping in mind that the specimen is several times thicker than the QD lateral size. We take $\varepsilon_{y y}=\varepsilon_{x x}$ and $\varepsilon_{y z}=\varepsilon_{x z}$ keeping $\varepsilon_{x y}=0$. While $\varepsilon_{x y}=0$ is a reasonable assumption since it is large at the heteroboundary, which is unsharp in the annealed QDs, other assumptions emerge as a value judgement. Note, that in $(\mathrm{In}, \mathrm{Ga}) \mathrm{As} / \mathrm{GaAs}$ QDs, there is a small inequivalence of [110] and [110] directions revealing in the exciton fine-structure splitting ${ }^{46}$ that can be changed in strained QDs, as advanced atomistic modeling shows. ${ }^{66,67}$

The strain mapping is performed by means of two methods, the geometric-phase analysis (GPA) ${ }^{59}$ and a modified peakpair analysis (PPA $)^{64}$ for verification. The GPA allows us to extract the crystal-lattice distortions locally at each atomic column with respect to the unstrained lattice area. The basic idea of such an analysis is based on the fast Fourier transform of the real-space image into the reciprocal space. Local strain tensor components, symmetric and rotation parts of distortion are calculated by derivation of the displacement obtained from two non-collinear Fourier components. ${ }^{64}$ The phase component of this function, called the geometric phase, describes the position-dependent lattice deviation with respect to a reference. The reference area of the crystal lattice is taken in the barrier region $40 \mathrm{~nm}$ away of the QD where the In concentration is negligibly small. The GPA strain-tensor components are extracted as

$$
\hat{\varepsilon}^{\mathrm{GPA}}=-\frac{1}{2 \pi} \mathbf{G}^{-1} \nabla \Phi_{g}(\mathbf{r}),
$$

where $\mathbf{G}=\left[\begin{array}{lll}g_{1 x} & g_{1 z} \\ g_{2 x} & g_{2 z}\end{array}\right]$ is the matrix formed by the components of two non-collinear reflexes $\mathbf{g}_{1}$ and $\mathbf{g}_{2}$, each of which is connected with the position-dependent geometric phase, $\Phi_{g}(\mathbf{r})$, as $\Phi_{g}(\mathbf{r})=2 \pi \Delta \mathbf{g}(\mathbf{r}) \cdot \mathbf{r}$, where $\mathbf{g}(\mathbf{r})$ represents the periodicities corresponding to the Bragg reflections. It has the following relationship with the lattices fringe spacing: $d=1 /|\mathbf{g}|$.

Additionally, the same STEM image with a single QD is processed using the PPA. In contrast to the GPA, the PPA is a real space procedure for strain mapping. PPA works with images having well-resolved fringe patterns, finding pairs of peaks along a preselected direction and distance in the affine transformed space defined by a pair of basis vectors $\mathbf{a}=\left(a_{x}, a_{y}\right)$ and $\mathbf{b}=\left(b_{x}, b_{y}\right)$. When the reference vectors are chosen on the filtered image, they can be used to construct an affine transformation. The next step in the PPA is the identification of peak-pairs using the chosen basis vectors and the intensity maxima set in the image. The Cauchy-Green strain components can be calculated by solving the following set of linear equations

$$
\begin{aligned}
& {\left[\begin{array}{l}
\varepsilon_{x x} \\
\varepsilon_{x z}
\end{array}\right]^{\mathrm{PPA}}=\left[\begin{array}{ll}
a_{x} & a_{z} \\
b_{x} & b_{z}
\end{array}\right]^{-1}\left[\begin{array}{l}
u_{x} \\
v_{x}
\end{array}\right],} \\
& {\left[\begin{array}{l}
\varepsilon_{z x} \\
\varepsilon_{z z}
\end{array}\right]^{\mathrm{PPA}}=\left[\begin{array}{ll}
a_{x} & a_{z} \\
b_{x} & b_{z}
\end{array}\right]^{-1}\left[\begin{array}{l}
u_{z} \\
v_{z}
\end{array}\right],}
\end{aligned}
$$

with coordinates of the displacements $\left(u_{x}, u_{y}\right)$ and $\left(v_{x}, v_{y}\right)$ with respect to the reference vectors $\mathbf{a}$ and $\mathbf{b}$.

The methods described above are based on the same general assumption that the relation between the atomic column positions in real crystal and features in the STEM image is 


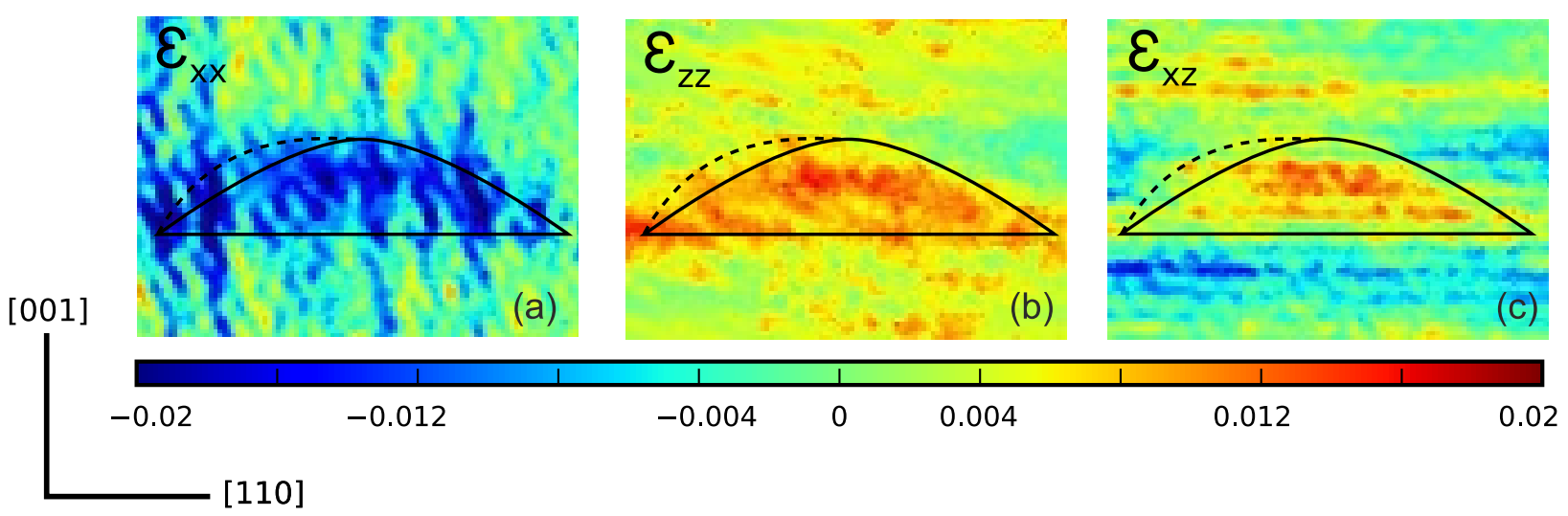

Figure 4. (Color online) Cauchy-Green strain tensor components extracted from the GPA analysis of HAADF-STEM images of a single QD, from left to right: (a) $\varepsilon_{x x}$, (b) $\varepsilon_{z z}$, and (c) $\varepsilon_{x z}$.

constant inside the studied region, where the phase shift between maxima and atoms is supposed to be constant. Both of these two methods, within the calculation error, gave identical results for all three tensor components $\varepsilon_{x x}, \varepsilon_{z z}$, and $\varepsilon_{x z}$ for the single QDs under study. The PPA requires less amount of memory and calculation time, given that the two-dimensional complex Fourier transform is not required. On the other hand, PPA fails when lattice peaks are not easily detected due to lower resolution of the image and appearance of sublattices due to a structure defect. In this case, filtering of the original image can be performed. Summarizing, we consider that both algorithms, GPA and PPA are useful for strain mapping, each having different advantages and limitations, and should be considered in each particular case specifically. In this work, we present the results of GPA only.

In general, the strain is measured with respect to the bulk lattice parameters of the material. Following this definition, the GPA(PPA) strain is connected with the material strain, $\varepsilon_{i j}$, as follows

$$
\left(1+\varepsilon_{i j}^{\mathrm{GPA}(\mathrm{PPA})}(\mathbf{r})\right)=\left(1-\varepsilon_{i j}(\mathbf{r})\right) \frac{a^{(\mathrm{In}, \mathrm{Ga}) \mathrm{As}}(\mathbf{r})}{a^{\mathrm{GaAs}}} .
$$

Here, the position-dependent lattice constant is determined by taking into account the concentration dependence of the solid compound via Vegard's law, $a^{(\mathrm{In}, \mathrm{Ga}) \mathrm{As}}(\mathbf{r})=a^{\mathrm{GaAs}} \cdot(1-c(\mathbf{r}))+$ $a^{\text {InAs }} \cdot c(\mathbf{r})$, where $c(\mathbf{r})$ is the position-dependent In concentration shown in Fig. 3.

Figure 4 illustrates the components of the physical strain, $\varepsilon_{x x}, \varepsilon_{z z}$, and $\varepsilon_{x z}$ evaluated from GPA analysis. The straintensor components extracted from high-resolution STEM images are averaged over the sample thickness, i.e., their values in the QD would be slightly larger in magnitude. Note, that an additional source of strain release arises from the sample thinning down to less than a $\mu \mathrm{m}$. In this case, the QD becomes closer to a surface than a deep-in-bulk QD. Therefore, both these effects should be considered as a source of measurement error for further evaluation of the results.

The negative value of $\varepsilon_{x x}$ shows that the crystal lattice is compressed in $x$ direction ([110]) [see fig. 4 (a)]. On the contrary, the $\varepsilon_{z z}$ component is mostly positive in the upper left region of the QD (see fig. 4 (b)), i.e., there is a stretching of the lattice in the growth direction $z$ ([001]). Fig. 4(c) shows a shear-strain $\varepsilon_{x z}$, which is present in a relatively small area having a mostly positive value and some periodicity in its distribution. In the wetting layer, the values of shear strain are predominantly negative, while inside the QDs this deformation is positive. Overall, the obtained results qualitatively well coincide with the finite-element modelling, represented previously in Ref. 45.

\section{ANALYSIS OF STRAIN-INDUCED QUADRUPOLE INTERACTION}

The change of energy experienced by the nuclear spin $\hat{\mathbf{I}}$ can be dependent of nuclear orientation. The charge environment of the nuclei interacts with the external electric potential $V$.

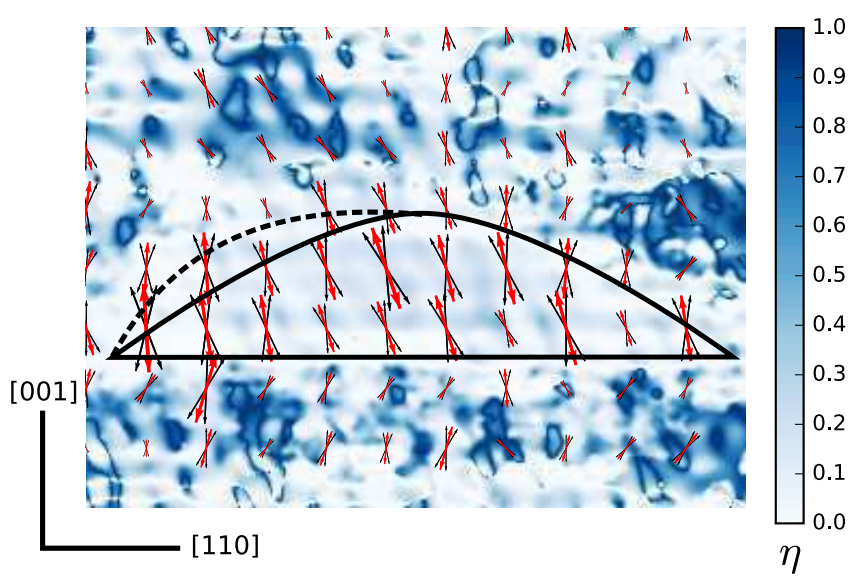

Figure 5. (Color online) EFG averaged over the propagation direction of the electron beam. The red arrows indicate the relative value of the strain-induced quadrupole frequency. All the microscopy data errors are indicated by the additional black arrows. The surface plot shows the spatial distribution of the EFG asymmetry $\eta$. Black lines indicate the shape of the QD extracted from the (In, Ga)As alloy concentration map. 

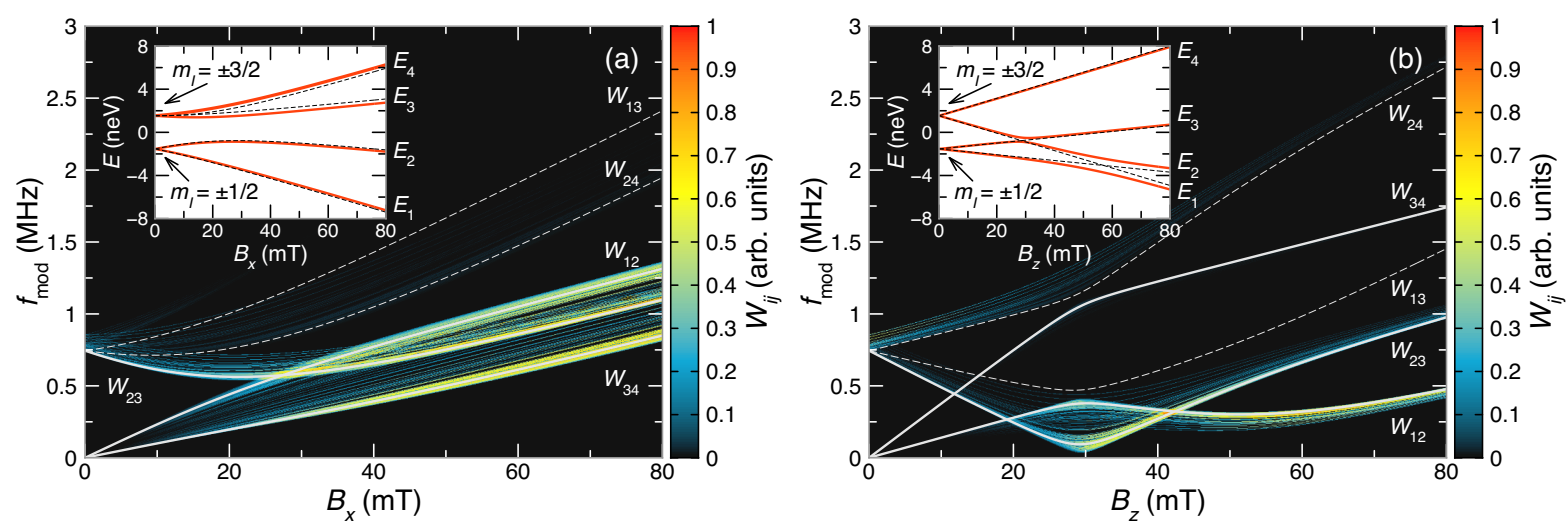

Figure 6. (Color online) The field-frequency maps of the nuclear spin-flip transition rates given by Eq. (6) calculated when the magnetic field is oriented perpendicular (a) or parallel (b) to the QD growth axis. The raster map indicates the probability of the transitions for ${ }^{71} \mathrm{Ga}$ nuclei with spin $I=3 / 2$ (as an example). The transitions between dipole-allowed and dipole forbidden states calculated with $\eta$ and $V_{\mathcal{Z} Z}$ over the QD volume are marked by solid and dashed lines, respectively. The inset graphs demonstrate the splittings of the nuclear-spin states for a single nucleus affected by a mean $v_{Q}$ in symmetric (dashed lines) or asymmetric (solid lines) quadrupole configurations.

In the equilibrium, the nuclei experience zero average electric field but nuclei having $I>1 / 2$ have also nonzero quadrupole moment interacting with the EFG denoted hereafter as $V_{i j} .68,69$ The physical origin of nonzero $V_{i j}$ is any inhomogeneity of the electric fields. The strongest effect is caused by the substitutional atoms in the alloy. Due to different electronegativity of the cation atoms, the anion As nuclei are highly affected by the EFG caused by the charge environment redistribution. For example, $5 \%$ electronegativity difference between the groupIII atoms leads to the nuclear-spin splitting of several tens of $\mathrm{MHz}$, as shown in (Al,Ga)As bulk. ${ }^{1}$

In self-assembled QDs, a weaker splitting would be obtained due to the crystal-lattice biaxial strain of several percent, leading to the quadrupole splitting of nuclear-spin states of the order of a MHz. ${ }^{43}$ Even in the absence of both effects, a strong electron localization would lead to the additional inhomogeneity of spin splitting caused by interaction with the applied electric field $V_{i j}{ }^{70}$ Our estimation shows, however, that the EFG variation due to inhomogeneity of the ground-state electron density inside the model QD leads to, at least, an order of magnitude smaller variation of spin splitting than its strain-induced value. However, in electrically driven QDs or in self-assembled QDs with a bias-controlled charge state, the electric-field induced EFG variation requires additional verification, as shown previously in a quantum well system. ${ }^{71}$

Considering further the strain-induced quadrupole interaction, the Hamiltonian reads as

$$
\hat{\mathcal{H}}=-\hbar \gamma_{I} \hat{\mathbf{I}} \cdot \mathbf{B}+\frac{h v_{Q}}{2}\left(3 \hat{I}_{\mathcal{Z}}^{2}-\hat{\mathbf{I}}^{2}+\frac{\eta}{2}\left(\hat{I}_{\mathcal{X}}^{2}+\hat{I}_{y}^{2}\right)\right) .
$$

Here, the first term couples nuclear spin $\hat{\mathbf{I}}$ of the $I$ th nucleus having the gyromagnetic ratio $\hbar \gamma_{I}$ with the external magnetic field $\mathbf{B}$. The second term describes the nuclear quadrupole interaction with strength $h v_{Q}$. The coordinate system $(\mathcal{X}, \mathcal{Y}, \mathcal{Z})$ corresponds to the major frame of the EFG acting on the nucleus generally not coinciding with the $(x, y, z)$ frame of the crystal. The quadrupole frequency $v_{Q}$ is proportional to the major EFG component $V_{\mathcal{Z} Z}$ satisfying $V_{\mathcal{Z} Z}>V_{y y}>V_{X X}$. The quadrupole asymmetry parameter, $\eta=\left(V_{x x}-V_{y y}\right) / V_{z z}$, is determined by the relative ratio of its other principal components.

The microscopic analysis allows to quantitatively evaluate the EFG tensor and, as a consequence, to map the quadrupole interaction over the sketch of the QD. The EFG tensor relates to the elastic strain as $V_{i j}=S_{i j k m} \varepsilon_{k m}$, where $S_{i j k m}$ is the fourth-rank gradient elastic tensor. ${ }^{72}$ Using the straintensor components extracted from HAADF-STEM analysis (see Fig. 4), we calculate all non-zero components of $V_{i j}$, and, correspondingly

$$
h v_{Q}=\frac{e Q V_{Z Z}}{4 I(2 I-1)}
$$

where $Q$ is the nuclear quadrupole moment. The results of such an analysis for ${ }^{71} \mathrm{Ga}$ are plotted in Fig. 5. The magnitude of $v_{Q}$ varies by the order of magnitude over the QD volume. However, the principal direction $\mathcal{Z}$ of the EFG varies by approximately 10 degrees, keeping a mean value misalignment of 8 degrees from the [001] crystal axis (see Fig. 5). The variation of the EFG asymmetry $\eta$ is found to be less than 0.2 over the whole QD volume.

To quantitatively evaluate the NMR absorption, the eigenvalue decomposition of Eq. (4), taking into account the spatial variation of $v_{Q}$ and $\eta$, is made. The spin-flip rates between $|i\rangle$ and $|j\rangle$ eigenstates having energies $E_{i}$ and $E_{j}$ are expressed as follows ${ }^{69}$

$$
W_{i j}=\frac{2 \pi}{\hbar}\left|\left\langle i\left|\hat{I}_{z}\right| j\right\rangle\right|^{2} \delta\left(E_{i}-E_{j}-h f_{\text {mod }}\right)
$$

when the $z$ axis coincides with the quantization axis of electron-spin observable. The transitions can be induced by either the absorption of radio-frequency electromagnetic fields or by the temporal variation of the Knight field due to electron-spin pumping, both modulated at frequency $f_{\text {mod }}$. The eigenstates $|i\rangle$ and $|j\rangle$ are no more eigenstates of $\hat{I}_{z}$, therefore the transitions with momentum projection by $\Delta m_{I}>1$ 
are allowed under certain conditions. ${ }^{73}$ The transition rates are calculated when scanning the magnitude of the external magnetic field oriented perpendicular [Fig. 6(a)] or parallel [Fig. 6(b)] to the [001] crystallographic axis, respectively. The statistics caused by the inhomogeneous variation of $v_{Q}$ and $\eta$ shown in Fig. 5 over the QD is taken into account here.

In both orientations of the magnetic field, the transition lines are strongly broadened due to inhomogeneity of the quadrupole interaction within the QD volume. A geometry with $\mathbf{B}$ aligned across the growth axis is more sensitive to the value of $v_{Q}$. Here, the Kramers doublets with $m_{I}= \pm 1 / 2$, $\pm 3 / 2$, etc., instantaneously mix in the $\mathbf{B}$ field, thus splitting the states linearly for $m_{I}= \pm 1 / 2$ and nonlinearly for higher states. The additional asymmetry of the quadrupole configuration given with $\eta>0$ or a small tilt of the magnetic field from exactly perpendicular to the EFG axis results in a change of the splitting, particularly for states having $\left|m_{I}\right|>1 / 2$. Both, the variation of $v_{Q}$ and $\eta$, and the magnetic-field tilting result in a broadening of the transition lines, as Fig. 6(a) shows. As a characteristic scale, the strongest transition between $m_{I}= \pm 1 / 2$ of ${ }^{71} \mathrm{Ga}$ spreads over about several hundred $\mathrm{kHz}$ in moderate fields $B>40 \mathrm{mT}$ [see Fig. 6(a)]. If the magnetic field is oriented along the EFG axis, the nuclearspin states are eigenstates of the Hamiltonian and do not mix by the magnetic field. However, a small tilt of the field results in a mixing, causing an anticrossing of lowest-energy $E_{1}$ and $E_{2}$ states [see inset in Fig. 6(b)]. Further mixing of states is provided by the asymmetric quadrupole configuration with $\eta>0$. Both effects lead to broadening of the sharp lines to values, however, smaller than in the perpendicular geometry by a factor of two, at least. This goes well with the recent observation of NMR lines in a single QD reported in Ref. 42 where the tail of the line of several hundred $\mathrm{kHz}$ is observed for all nuclear isotopes.

The shear strain expressed in the dot as $\varepsilon_{x z}$ and providing nonzero $\eta$ further modifies qualitatively the NMR spectra. For the sake of comparison, the energy-level schemes in both symmetric and asymmetric quadrupole configurations are shown in the insets of Figs. 6(a) and 6(b). The frequency shifts of several tens of $\mathrm{kHz}$ are observed in both geometries. In addition to that, the spin-level $E_{2}$ and $E_{3}$ anticrossing exists when $\eta>0$ [see Fig. 6(b)]. The sample tilting is a natural way to reduce the broadening, ${ }^{69}$ that works well in single-QD NMR. ${ }^{42}$ However, full compensation of the spin-level anticrossings is prohibited if $\eta \neq 0$, as demonstrated in Fig. 6(b).

\section{CONCLUSION}

To conclude, the microscopic study of the quadrupole interaction in self-assembled (In,Ga)As QD is done by using scanning transmission electron microscopy. The HAADF-STEM technique allows us to extract the (In,Ga)As alloy concentration of In and $\mathrm{Ga}$ atoms, keeping the atomic-column resolution of the microscopy image, and to evaluate the in-plane components of the Cauchy-Green strain tensor by using the geometric phase analysis. Further mapping of the biaxial and shear strain components allows for quantitative reconstruction of the strain-induced EFG tensor components. Modelling the NMR absorption spectra, the magnitudes of the NMR lines broadenings and a shift of certain NMR transitions caused by the asymmetry of the EFG tensor are evaluated. In particular, the asymmetry parameter of the quadrupole interaction leads to shifts and anticrossings of certain transitions in the NMR absorption spectrum.

\section{ACKNOWLEDGMENTS}

We would like to thank I.V. Ignatiev (Saint-Petersburg State University, Russia), A. Greilich, D.R. Yakovlev, and M. Bayer (Technical University of Dortmund, Germany) for the discussion and valuable comments on the paper. This work is partially supported by the Russian Foundation for Basic Research and the Deutsche Forschungsgemeinschaft in the frame of the International Collaborative Research Center TRR 160 and by the EU FET-program SPANGL4Q. P.S.S. would like to acknowledge support of SPbSU research grant No. 11.38.213.2014 and the BISIP Santander Scholarships programme (Bremen International Student Internship) whereby the microscopic experiments were conducted at the University of Bremen. M.Y.P. acknowledges support of the Russian Ministry of Education and Science under grant No. 11.G34.31.0067. A.D.W. acknowledges gratefully support of Mercur Pr-2013-0001, BMBF - Q.com-H 16KIS0109, and the DFH/UFA CDFA-05-06. K. M.-C. acknowledges support from the Deutsche Forschungsgemeinschaft (DFG) under contract No. MU 3660/1-1.
* The correspondence have to be sent to m.petrov@ spbu.ru

1 Optical Orientation, Eds. F. Meier and B. P. Zakharchenya (North-Holland, Amsterdam, 1984).

2 Spin Physics in Semiconductors, ed. by M. I. Dyakonov (SpringerVerlag, Berlin 2008).

3 G. Lampel, "Nuclear dynamic polarization by optical electronic and optical pumping in semiconductors", Phys. Rev. Lett. 20, 491 (1968).

4 M. I. Dyakonov and V. I. Perel, "Optical orientation in a system of electrons and lattice nuclei in semiconductors. Theory.", Zh. Eksp. Teor. Fiz. 65, 362 (1973) [Sov. Phys. JETP 38, 177 (1974)].
5 D. Gammon, E. S. Snow, B. V. Shanabrook, D. S. Katzer, and D. Park, "Homogeneous linewidths in the optical spectrum of a single Gallium Arsenide quantum dot", Science 273, 87 (1996).

${ }^{6}$ D. Gammon, Al. L. Efros, T. A. Kennedy, M. Rosen, D. S. Katzer, D. Park, S. W. Brown, V. L. Korenev and I. A. Merkulov, "Electron and nuclear spin interactions in the optical spectra of single GaAs quantum dots", Phys. Rev. Lett. 86, 5176 (2001).

7 I. A. Merkulov, Al. L. Efros, and M. Rosen, "Electron spin relaxation by nuclei in semiconductor quantum dots", Phys. Rev. B 65, 205309 (2002). 
8 A. V. Khaetskii, D. Loss, and L. Glazman, "Electron spin decoherence in quantum dots due to interaction with nuclei", Phys. Rev. Lett. 88, 186802 (2002).

9 W. A. Coish and D. Loss, "Hyperfine interaction in a quantum dot: Non-Markovian electron spin dynamics", Phys. Rev. B 70, 195340 (2004).

10 A. C. Johnson, J. R. Petta, J. M. Taylor, A. Yacoby, M. D. Lukin, C. M. Marcus, M. P. Hanson, and A. C. Gossard, "Triplet-singlet spin relaxation via nuclei in a double quantum dot", Nature (London) 435, 925 (2005).

11 F. H. L. Koppens, J. A. Folk, J. M. Elzerman, R. Hanson, L. H. Willems van Beveren, I. T. Vink, H. P. Tranitz, W. Wegscheider, L. P. Kouwenhoven, and L. M. K. Vandersypen, "Control and detection of singlet-triplet mixing in a random nuclear field", Science 309, 5739 (2005).

12 P.-F. Braun, X. Marie, L. Lombez, B. Urbaszek, T. Amand, P. Renucci, V. K. Kalevich, K. V. Kavokin, O. Krebs, P. Voisin, and Y. Masumoto, "Direct observation of the electron spin relaxation induced by nuclei in quantum dots", Phys. Rev. Lett. 94, 116601 (2005).

13 C. W. Lai, P. Maletinsky, A. Badolato, and A. Imamoglu, "Knightfield-enabled nuclear spin polarization in single quantum dots", Phys. Rev. Lett. 96, 167403 (2006).

14 P.-F. Braun, B. Urbaszek, T. Amand, X. Marie, O. Krebs, B. Eble, A. Lemaitre, and P. Voisin, "Bistability of the nuclear polarization created through optical pumping in $\mathrm{In}_{1-x} \mathrm{Ga}_{x}$ As quantum dots", Phys. Rev. B 74, 245306 (2006).

15 A. Greilich, A. Shabaev, D. R. Yakovlev, Al. L. Efros, I. A. Yugova, D. Reuter, A. D. Wieck, M. Bayer, "Nuclei-induced frequency focusing of electron spin coherence", Science 317, 1896 (2007).

16 P. Maletinsky, C. W. Lai, A. Badolato, and A. Imamoglu, "Nonlinear dynamics of quantum dot nuclear spins", Phys. Rev. B 75, 035409 (2007).

17 A. I. Tartakovskii, T. Wright, A. Russell, V. I. Fal'ko, A. B. Van'kov, J. Skiba-Szymanska, I. Drouzas, R. S. Kolodka, M S. Skolnick, P. W. Fry, A. Tahraoui, H.-Y. Liu, and M. Hopkinson, "Nuclear spin switch in semiconductor quantum dots", Phys. Rev. Lett. 98, 026806 (2007).

18 R. Oulton, A. Greilich, S. Yu. Verbin, R. V. Cherbunin, T. Auer, D. R. Yakovlev, M. Bayer, I. A. Merkulov, V. Stavarache, D. Reuter, and A. D. Wieck, "Subsecond spin relaxation times in quantum dots at zero applied magnetic field due to a strong electron-nuclear interaction”, Phys. Rev. Lett. 98, 107401 (2007).

19 S. G. Carter, A. Shabaev, S. E. Economou, T. A. Kennedy, A. S. Bracker, and T. L. Reinecke, "Directing nuclear spin flips in InAs quantum dots using detuned optical pulse trains", Phys. Rev. Lett. 102, 167403 (2009).

${ }^{20}$ X. Xu, W. Yao, B. Sun, D. G. Steel, A. S. Bracker, D. Gammon, and L. J. Sham, "Optically controlled locking of the nuclear field via coherent dark-state spectroscopy", Nature (London) 459, 1105 (2009).

${ }^{21}$ P. Maletinsky, M. Kroner, and A. Imamoglu, "Breakdown of the nuclear-spin-temperature approach in quantum-dot demagnetization experiments", Nat. Phys. 5, 407 (2009).

${ }^{22}$ C. Latta, A. Högele, Y. Zhao, A. N. Vamivakas, P. Maletinsky, M. Kroner, J. Dreiser, I. Carusotto, A. Badolato, D. Schuh, W. Wegscheider, M. Atature, and A. Imamoglu, "Confluence of resonant laser excitation and bidirectional quantum-dot nuclear-spin polarization", Nat. Phys. 5, 758 (2009).

23 I. T. Vink, K. C. Nowack, F. H. L. Koppens, J. Danon, Y. V. Nazarov, and L. M. K. Vandersypen, "Locking electron spins into magnetic resonance by electron-nuclear feedback", Nat. Phys. 5, 764 (2009).
${ }^{24}$ R. V. Cherbunin, S. Yu. Verbin, T. Auer, D. R. Yakovlev, D. Reuter, A. D. Wieck, I. Ya. Gerlovin, I. V. Ignatiev, D. V. Vishnevsky, and M. Bayer, "Dynamics of the nuclear spin polarization by optically oriented electrons in a (In, Ga)As/GaAs quantum dot ensemble", Phys. Rev. B 80, 035326 (2009).

25 O. Krebs, P. Maletinsky, T. Amand, B. Urbaszek, A. Lemaître, P. Voisin, X. Marie, and A. Imamoglu, "Anomalous Hanle effect due to optically created transverse Overhauser field in single InAs/GaAs quantum dots", Phys. Rev. Lett. 104, 056603 (2010).

${ }^{26}$ E. A. Chekhovich, M. N. Makhonin, K. V. Kavokin, A. B. Krysa, M. S. Skolnick, and A. I. Tartakovskii, "Pumping of nuclear spins by optical excitation of spin-forbidden transitions in a quantum dot", Phys. Rev. Lett. 104, 066804 (2010).

27 M. Issler, E. M. Kessler, G. Giedke, S. Yelin, I. Cirac, M. D. Lukin, and A. Imamoglu, "Nuclear spin cooling using Overhauser-field selective coherent population trapping", Phys. Rev. Lett. 105, 267202 (2010).

28 A. Högele, M. Kroner, C. Latta, M. Claassen, I. Carusotto, C. Bulutay, and A. Imamoglu, "Dynamic nuclear spin polarization in the resonant laser excitation of an InGaAs quantum dot", Phys. Rev. Lett. 108, 197403 (2012).

29 J. Puebla, E. A. Chekhovich, M. Hopkinson, P. Senellart, A. Lemaitre, M. S. Skolnick, and A. I. Tartakovskii, "Dynamic nuclear polarization in InGaAs/GaAs and GaAs/AlGaAs quantum dots under nonresonant ultralow-power optical excitation", Phys. Rev. B 88, 045306 (2013).

30 A. Imamoglu, E. Knill, L. Tian, and P. Zoller, "Optical pumping of quantum-dot nuclear spins”, Phys. Rev. Lett. 91, 017402 (2003).

31 H. Christ, J. I. Cirac, and G. Giedke, "Quantum description of nuclear spin cooling in a quantum dot”, Phys. Rev. B 75, 155324 (2007).

32 M. Yu. Petrov, G. G. Kozlov, I. V. Ignatiev, R. V. Cherbunin, D. R. Yakovlev, and M. Bayer, "Coupled electron-nuclear spin dynamics in quantum dots: A graded box model approach”, Phys. Rev. B 80, 125318 (2009).

33 J. Hildmann, E. Kavousanaki, G. Burkard, and H. Ribeiro, "Quantum limit for nuclear spin polarization in semiconductor quantum dots", Phys. Rev. B 89, 205302 (2014).

${ }^{34}$ M. N. Makhonin, E. A. Chekhovich, P. Senellart, A. Lemaître, M. S. Skolnick, and A. I. Tartakovskii, "Optically tunable nuclear magnetic resonance in a single quantum dot", Phys. Rev. B 82, 161309(R) (2010).

35 K. Flisinski, I. Ya. Gerlovin, I. V. Ignatiev, M. Yu. Petrov, S. Yu. Verbin, D. R. Yakovlev, D. Reuter, A. D. Wieck, and M. Bayer, "Optically detected magnetic resonance at the quadrupole-split nuclear states in (In,Ga)As/GaAs quantum dots", Phys. Rev. B 82, 081308(R) (2010).

36 E. A. Chekhovich, K. V. Kavokin, J. Puebla, A. B. Krysa, M. Hopkinson, A. D. Andreev, A M. Sanchez, R. Beanland, M. S. Skolnick, and A. I. Tartakovskii, "Structural analysis of strained quantum dots using nuclear magnetic resonance", Nat. Nanotechnol. 7, 646 (2012).

37 M. Munsch, G. Wüst, A. V. Kuhlmann, F. Xue, A. Ludwig, D. Reuter, A. D. Wieck, M. Poggio, and R. J. Warburton, "Manipulation of the nuclear spin ensemble in a quantum dot with chirped magnetic resonance pulses", Nat. Nanotechnol. 9, 671 (2014).

38 Y. Li, N. Sinitsyn, D. L. Smith, D. Reuter, A. D. Wieck, D. R. Yakovlev, M. Bayer, and S. A. Crooker, Phys. Rev. Lett. 108, 186603 (2012).

39 F. Berski, J. Hübner, M. Oestreich, A. Ludwig, A. D. Wieck, and M. Glazov, "Interplay of electron and nuclear spin noise in GaAs" (unpublished); arXiv:1506.05370.

40 P. Peddibhotla, F. Xue, H. I. T. Hauge, S. Assali, E. P. A. M. Bakkers, and M. Poggio, "Harnessing nuclear spin polarization 
fluctuations in a semiconductor nanowire”, Nat. Phys. 9, 631 (2013).

41 C. Bulutay, "Quadrupolar spectra of nuclear spins in strained $\mathrm{In}_{x} \mathrm{Ga}_{1-x}$ As quantum dots", Phys. Rev. B 85, 115313 (2012).

42 C. Bulutay, E. A. Chekhovich, and A. I. Tartakovskii, "Nuclear magnetic resonance inverse spectra of InGaAs quantum dots: Atomistic level structural information", Phys. Rev. B 90, 205425 (2014).

${ }^{43}$ R. I. Dzhioev and V. L. Korenev, "Stabilization of the electronnuclear spin orientation in quantum dots by the nuclear quadrupole interaction”, Phys. Rev. Lett. 99, 037401 (2007).

${ }^{44}$ R. V. Cherbunin, K. Flisinski, I. Ya. Gerlovin, I. V. Ignatiev, M. S. Kuznetsova, M. Yu. Petrov, D. R. Yakovlev, D. Reuter, A. D. Wieck, and M. Bayer, "Resonant nuclear spin pumping in (In,Ga)As quantum dots”, Phys. Rev. B 84, 041304 (2011).

45 M. S. Kuznetsova, K. Flisinski, I. Ya. Gerlovin, M. Yu. Petrov, I. V. Ignatiev, S. Yu. Verbin, D. R. Yakovlev, D. Reuter, A. D. Wieck, and M. Bayer, "Nuclear magnetic resonances in (In,Ga)As/GaAs quantum dots studied by resonant optical pumping", Phys. Rev. B 89, 125304 (2009).

46 W. Langbein, P. Borri, U. Woggon, V. Stavarache, D. Reuter, and A. D. Wieck, "Control of fine-structure splitting and biexciton binding in $\operatorname{In}_{x} \mathrm{Ga}_{1-x}$ As quantum dots by annealing", Phys. Rev. B 69, 161301(R) (2004).

${ }^{47}$ M. Yu. Petrov, I. V. Ignatiev, S. V. Poltavtsev, A. Greilich, A. Bauschulte, D. R. Yakovlev, and M. Bayer, "Effect of thermal annealing on the hyperfine interaction in InAs/GaAs quantum dots", Phys. Rev. B 78, 045315 (2008).

48 The analyzing sample was exposed to a rapid thermal annealing of $30 \mathrm{~s}$ duration at temperature $T_{a}=920{ }^{\circ} \mathrm{C}$. As a result of the bandgap renormalization, the ground state photoluminescence changes from $1.06 \mathrm{eV}$ to $1.37 \mathrm{eV}$.

49 A. Rosenauer, Transmission Electron Microscopy of Semiconductor Nanostructures: An Analysis of Composition and Strain State, (Springer-Verlag, Berlin, 2003).

50 A. Rosenauer, T. Mehrtens, K. Müller, K. Gries, M. Schowalter, S. Bley, P. V. Satyam, A. Avramescu, K. Engl, and S. Lutgen, "2Dcomposition mapping in InGaN without electron beam induced clustering of indium by STEM HAADF Z-contrast imaging”, J. Phys. Conf. Ser. 326, 012040 (2011).

${ }^{51}$ H. Kauko, T. Grieb, R. Bjørge, M. Schowalter, A. M. Munshi, H. Weman, A. Rosenauer, and A. T. J. van Helvoort, "Compositional characterization of $\mathrm{GaAs} / \mathrm{GaAsSb}$ nanowires by quantitative HAADF-STEM", Micron 44, 254 (2013).

52 T. Grieb, K. Müller, O. Rubel, R. Fritz, C. Gloistein, N. Neugebohrn, M. Schowalter, K. Volz, and A. Roseanuer, "Determination of Nitrogen Concentration in Dilute GaNAs by STEM HAADF ZContrast Imaging”, J. Phys. Conf. Ser. 326, 012033 (2011).

53 T. Grieb, K. Müller, R. Fritz, V. Grillo, M. Schowalter, K. Volz, and A. Rosenauer, "Quantitative chemical evaluation of dilute GaNAs using ADF STEM: Avoiding surface strain induced artifacts", Ultramicroscopy 129, 1 (2013).

54 T. Grieb, K. Müller, R. Fritz, M. Schowalter, N. Neugebohrn, N. Knaub, K. Volz, and A. Rosenauer, "Determination of the chemical composition of GaNAs using STEM HAADF imaging and STEM strain state analysis", Ultramicroscopy 117, 15 (2012).

55 A. Rosenauer, K. Gries, K. Müller, A. Pretorius, M. Schowalter, A. Avramescu, K. Engl, and S. Lutgen, "Measurement of specimen thickness and composition in $\mathrm{Al}_{x} \mathrm{Ga}_{1-x} \mathrm{~N} / \mathrm{GaN}$ using highangle annular dark field images", Ultramicroscopy 109, 1171 (2009).

56 T. Mehrtens, M. Schowalter, D. Tytko, P. Choi, D. Raabe, L. Hoffmann, H. Jönen, U. Rossow, A. Hangleiter and A. Rosenauer,
"Measurement of the indium concentration in high indium content InGaN layers by scanning transmission electron microscopy and atom probe tomography”, Appl. Phys. Lett. 102, 132112 (2013).

57 T. Mehrtens, K. Müller, M. Schowalter, D. Hu, D. M. Schaadt, and A. Rosenauer, "Measurement of indium concentration profiles and segregation efficiencies from high-angle annular dark field-scanning transmission electron microscopy images", Ultramicroscopy 131, 1 (2013).

58 V. Grillo, K. Mueller, K. Volz, F. Glas, T. Grieb and A. Rosenauer, "Strain, composition and disorder in ADF imaging of semi- conductors", J. Phys. Conf. Ser. 326, 012006 (2011).

59 M. J. Hÿtch, E. Snoeck, and R. Kilaas, "Quantitative measurement of displacement and strain fields from HREM micrographs", Ultramicroscopy 74, 131 (1998).

${ }^{60}$ N. Cherkashin, S. Reboh, M. J. Hÿtch, A. Claverie, V. V. Preobrazhenskii, M. A. Putyato3 B. R. Semyagin, and V. V. Chaldyshev, "Determination of stress, strain, and elemental distribution within In $(\mathrm{Ga})$ As quantum dots embedded in GaAs using advanced transmission electron microscopy", Appl. Phys. Lett. 102, 1731115 (2013).

61 A. E. Frazho and W. Bhosri, "An Operator Perspective on Signals and Systems", (Operator Theory Advances and Applications, Vol. 204, 283 (2010).

${ }^{62}$ STEMsim program.

${ }^{63}$ D. V. Sridhara Rao, R. Sankarasubramanian, K. Muraleedharan, T. Mehrtens, A. Rosenauer and D. Banerjee, "Quantitative strain and compositional studies of $\mathrm{In}_{x} \mathrm{Ga}_{1-x}$ As epilayer in a GaAsbased pHEMT device structure by TEM techniques", Microsc. Microanal. 20, 1262 (2014).

${ }^{64}$ P. L. Galindo, S. Kret, A. M. Sanchez, J. Y. Laval, A. Yáñez, J. Pizarro, E. Guerrero, T. Ben, and S. I. Molina, "The peak pairs algorithm for strain mapping from HRTEM images", Ultramicroscopy 107, 1186 (2007).

${ }^{65}$ D. L. Sales, J. Pizarro, P. L. Galindo, R. Garcia, G. Trevisi, P. Frigeri, L. Nasi, S. Franchi and S. I. Molina, "Critical strain region evaluation of self-assembled semiconductor quantum dots", Nanotechnology 18, 475503 (2007).

66 R. Singh and G. Bester, "Lower bound for the excitonic fine structure splitting in self-assembled quantum dots", Phys. Rev. Lett. 104, 196803 (2010).

${ }^{67}$ R. Singh and G. Bester, "Electronic and optical properties of strained $\mathrm{In}_{x} \mathrm{Ga}_{1-x} \mathrm{As} / \mathrm{GaAs}$ and strain-free $\mathrm{GaAs} / \mathrm{Al}_{0.3} \mathrm{Ga}_{0.7} \mathrm{As}$ quantum dots on (110) substrates", Phys. Rev. B 88, 075430 (2013).

68 A. Abragam, Principles of Nuclear Magnetism (Oxford University Press, London, 1962).

${ }^{69}$ C. P. Slichter, Principles of Magnetic Resonance, 3rd ed. (Springer-Verlag, Berlin, 1992).

${ }^{70}$ E.Brun, R. J. Mahler, H. Mahon, and W. L. Pierce, "Electrically Induced Nuclear Quadrupole Spin Transitions in a GaAs Single Crystal", Phys. Rev. 129, 1965 (1963).

${ }^{71}$ M. Eickhoff, B. Lenzmann, D. Suter, S. F. Hayes, and A. D. Wieck, "Mapping of strain and electric fields in $\mathrm{GaAs} / \mathrm{Al}_{x} \mathrm{Ga}_{1-x} \mathrm{As}$ quantum-well samples by laser-assisted NMR", Phys. Rev. B 67, 085308 (2003).

72 R. K. Sundfors, "Experimental gradient-elastic tensors and chemical bonding in III-V semiconductors", Phys. Rev. B 10, 4244 (1974)

${ }^{73}$ M. Eickhoff, B. Lenzmann, G. Flinn, and D. Suter, "Coupling mechanisms for optically induced NMR in GaAs quantum wells", Phys. Rev. B 65, 125301 (2002). 